\title{
An Application of Hydrogen Thermophysical Properties Database "All in One Live CD"
}

\author{
S. Momoki ${ }^{1}$, O. Jambal ${ }^{1}$, T. Yamaguchi ${ }^{1}$, R. Akasaka ${ }^{2}$, Y. Takata $^{3}$ \\ 1. Graduate School of Science and Technology, Nagasaki University, Nagasaki, Japan \\ 2. Department of Mechanical Engineering, Kyushu Sangyo University, Fukuoka, Japan \\ 3. Department of Mechanical Engineering, Kyushu University, Fukuoka, Japan \\ Corresponding author: S. Momoki e-mail: momoki@nagasaki-u.ac.jp
}

\begin{abstract}
We are developing a database for the thermophysical properties of hydrogen that supports a wide range of parameters including high pressures and high temperatures. The database performance requirements were studied and, in this paper, we propose an application that combines a server-client database and a live CD. For this, we have developed an "All in One Live CD" application. Web interfaces provide excellent user interfaces for databases. However, there are some disadvantages for web interfaces related to server maintenance and access restrictions. Our new application, the "All in One Live CD" is free of these disadvantages, and grants a database with a property estimation service regardless of the computer environment. From a single boot via the "All in One Live CD", the database becomes accessible on multiple computers so that this feature makes the new application a unique solution as a distribution media.
\end{abstract}

Keywords fluid; high pressures; high temperatures; hydrogen; live CD; thermophysical properties database

\section{Introduction}

We are developing a database for the thermophysical properties of hydrogen within the framework of projects carried out by HYDROGENIUS, the Research Center for Hydrogen Industrial Use and Storage [1], which was founded under the Japanese National Institute of Advanced Industrial Science and Technology. The demands of establishing the safety technology of hydrogen utilization have resulted in efforts to satisfy the needs for the accurate thermophysical properties over a wide range of parameters.

We are aiming to develop a hydrogen thermophysical properties database that is complete and accessible on the internet from all over the world. Its core component is a PROPATH-style database. Since 1984, PROPATH [2] has provided a service in estimating the properties of various fluids in the style that the calculated properties are given in accordance with certain thermodynamic processes. There are many general purpose databases that offer calculation services for the thermophysical properties of fluids. Among these databases, the NIST Reference Fluid Thermodynamic and Transport Properties Database (REFPROP) [3] and NIST Chemistry WebBook [4] support a standard formulation of the thermophysical properties of hydrogen. In order to establish a reliable analysis and design, we also need to know the thermophysical properties of hydrogen at high pressures and high temperatures. Sakoda et al. have conducted a detailed review [5] on the available thermophysical properties of hydrogen and they have shown that, for high pressure and high temperature conditions, the experimental data required for the formulation of the prediction equations is scarce while it is crucial to provide the necessary data.

The primary objective of our database is to provide reliable and complete thermophysical properties of hydrogen for a wide range of parameters that include high pressures and high temperatures. In the context of studying the technology for the safe utilization of hydrogen, our thermophysical properties team is engaged in measuring the various properties of hydrogen, such as density, viscosity, sound velocity, thermal conductivity, and specific heat, under the conditions of $100 \mathrm{MPa}$ and $500{ }^{\circ} \mathrm{C}$. At present, we have developed a preliminary database, which includes an estimation service based on currently available equations. We are examining the database performance requirements and this paper describes the result of our investigation, which is an application that can be used for any database. 
As a part of this ongoing project, we have created the application the "All in One Live CD". This live $C D$ makes the database and the thermophysical property estimation service available regardless of the computer environment. Once booted from "All in One Live CD", the computer acts as a database server and, as this application integrates into the existing local network environment, other users on the same local network can share the database.

\section{Development of a Database for Hydrogen Thermophysical Properties}

\subsection{Calculation Service and Target Properties}

In the design and analyses, engineers often encounter the need to make calculations for thermophysical properties of the working fluids at corresponding conditions of the processes. For pure fluids, there are approximately 30 technologically significant properties [6], and reliable thermophysical properties data are necessary to advance the basic science of hydrogen and to improve the standardization of engineering applications. We aim to integrate presently available information about hydrogen, including the published investigation results, and to provide active components so that the database would interface to computational services for a wide range of parameters. The availability and assessment of data on the thermophysical properties of hydrogen have recently been surveyed and compiled by the research group at the Idaho University. Jacobsen et al. [7] and Leachman et al. [8] have recommended that new additional experimental data are needed, especially for an increased temperature range.

Presently, the thermophysical properties team of the HYDROGENIUS is conducting measurements of the density, the coefficient of viscosity, the isobaric specific heat, the sound velocity, and the solubility of hydrogen at high temperatures and high pressures. Formulation and validation of equations of state require extensive and accurate experimental data, and our database system is intended to be an effective tool to provide hydrogen properties based on the equations of state. Figure 1 illustrates the correlation of thermophysical properties. The properties listed in the lower left and right boxes represent the thermophysical properties

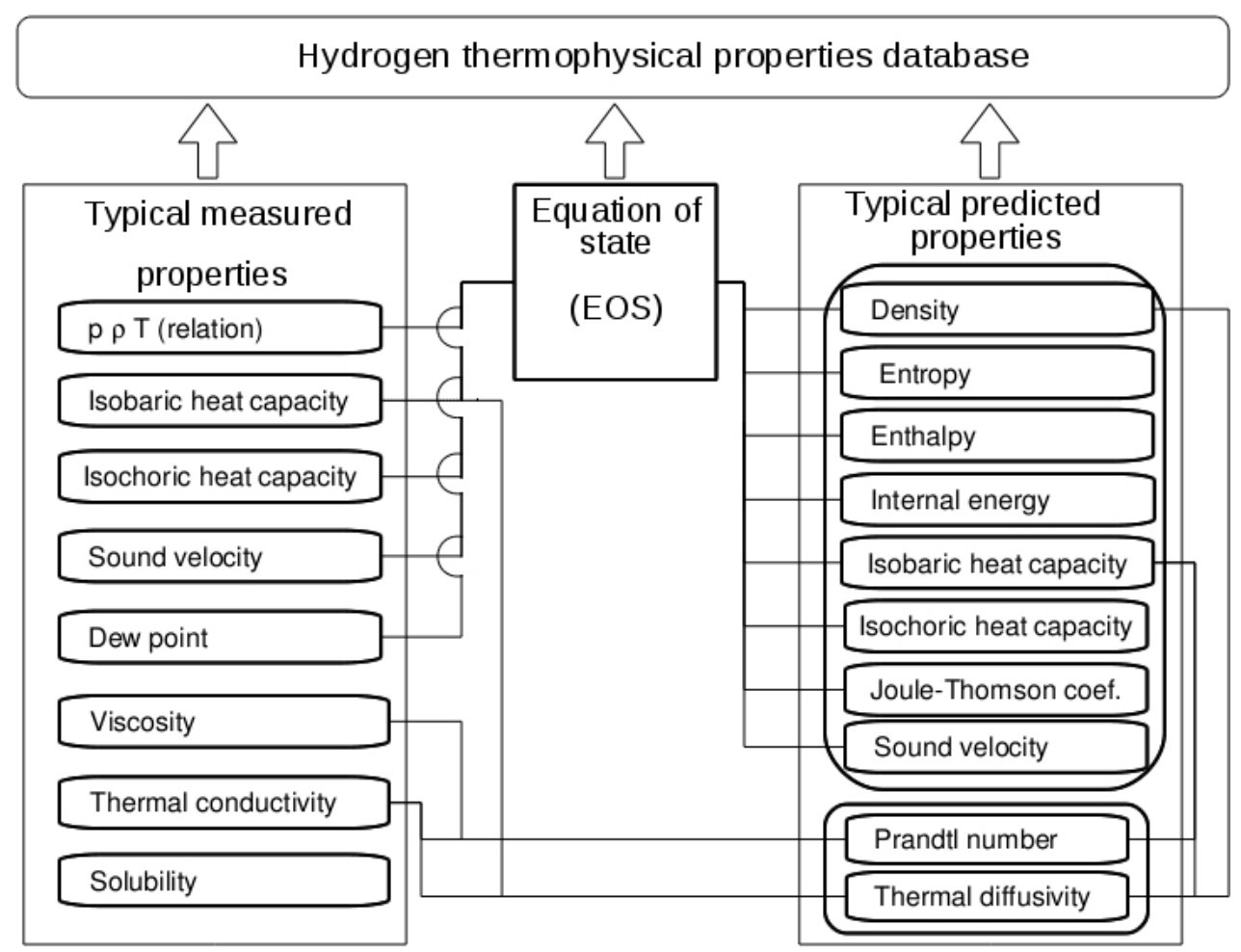

Fig. 1 Thermophysical properties and equation of state 
supported by our database. The left box contains the list of representatives of the experimentally accessible properties and the right box is for the properties that are calculated. Equation of state in the central box is an essential part of our database since supporting a calculation service is the most important feature of the database. At the moment, presently available equations of state [9]-[12] are applied in the database while a new equation of state is being formulated at the HYDROGENIUS. Equation of state relates the various thermodynamic properties to one another. These interrelations and the correlation of transport properties are shown by the lines in Fig. 1. Some thermodynamic properties (such as density, isobaric heat capacity, sound velocity, etc) can be both measured and predicted. This allows ascertaining the availability of precise equations of state, as validation is carried out on the basis of the experimental data and of the values generated from the equation.

In offering the estimation of thermophysical properties, we took into account that there are specific demands connected with design and analysis. In design and analysis, it is necessary to calculate the thermophysical properties repeatedly at different combinations of independent properties. Therefore, it is efficient to provide predictions for such combinations of properties, and PROPATH-style databases are capable to offering many sets of combinations encountered in thermodynamic processes. These sets of combinations can be grouped in accordance with the certain states that occur in engineering applications. Our database is concerned with the vaporliquid equilibrium state properties for a given pressure and for a given temperature, heated steam and compressed liquid properties at given pressure and temperature, the properties in correlation with various thermodynamic processes and miscellaneous properties such as specific points of state. The current database uses existing PROPATH templates for the sets of thermophysical properties. Moreover, new sets are being considered for the calculation of hydrogen related design and analysis. Presently, a set to predict the temperature, entropy and volume for the given pressure and enthalpy has been added as this set of properties combination is repeatedly encountered for hydrogen compressor calculations. The target properties of the database are density, enthalpy, entropy, internal energy, isobaric heat capacity, isochoric heat capacity, the Joule Thomson coefficient, sound velocity, the Prandtl number, thermal diffusivity, latent heat of vaporization, surface tension, thermal conductivity, viscosity, the isentropic exponent, the Laplace coefficient, ion product, volumetric coefficient of expansion, isentropic compressibility, isothermal compressibility, pressure coefficient, as well as other relevant properties.

\subsection{Web-I nterface of the Database}

Our database is intended to be an open internet accessible source of information for hydrogen thermophysical properties. All information in the database will be accessible through a web interface and once accessed, the database can act as a calculation server. The database is under development and our database project team web page [13] includes part of the outcome of the present work. The web interface to the database uses a standard protocol for exchanging information over the network. Because this provision is available to anyone having a web browser, the user does not need to install any software related to the database. Through the database web interface, the user can perform the predictions of hydrogen thermophysical properties and obtain the published experimental data including the general information related to the equations of state mainly related to the published papers.

\section{I mplementation of the Database}

\subsection{Prediction of the Thermophysical Properties of Hydrogen}

At present, one major component of our database is the computer program that solves a set of equations of state for hydrogen. The aim of this prediction service is to provide a properties estimation tool in addition to other available data on hydrogen. Currently, the computer programs are completed for five available prediction equations, namely of Woolley [9], GERG [10], and Leachman [11] for normal-hydrogen and of Leachman [11] and Younglove [12] for para-hydrogen. 
Table 1 Upper limits of predictions

\begin{tabular}{|c|c|c|c|c|}
\hline $\begin{array}{l}\text { Upper limit of } \\
\text { temperature } \\
(\mathrm{K})\end{array}$ & $\begin{array}{l}\text { Upper limit of } \\
\text { pressure } \\
(\mathrm{MPa})\end{array}$ & Para-hydrogen & $\begin{array}{l}\text { Upper limit of } \\
\text { temperature } \\
(\mathrm{K})\end{array}$ & $\begin{array}{l}\text { Upper limit of } \\
\text { pressure (MPa) }\end{array}$ \\
\hline 600 & 170 & Leachman [1] & 1000 & 2000 \\
\hline 700 & 300 & Younglove [12] & 400 & 121 \\
\hline 1000 & 2000 & \multicolumn{2}{|c}{} \\
\hline
\end{tabular}

These equations have been chosen on the basis of a detailed review published elsewhere [5] together with comparisons of PVT, saturated properties, heat capacity, and sound velocity as well as a summary of data for the critical point, normal point and triple point values. In Table 1, the upper limits of temperature and pressure for the prediction equations are given.

The computer programs are menu driven, and the user has the option to choose the equation from a list of equations. For each of the equations, there are 24 independent sets of variable combinations of hydrogen properties. These 24 combinations can be classified into (1) vaporliquid equilibrium state properties for a given pressure, (2) vapor-liquid equilibrium state properties for a given temperature, (3) heated steam and compressed liquid properties at a given pressure and temperature, (4) thermophysical properties calculation in relation to thermodynamic processes, and (5) miscellaneous properties such as critical points of state. When a menu selection is made, our database pulls the corresponding stored data for performing the prediction and the user needs to enter the input value. The programs will prompt the user for the supporting data necessary to carry out the calculation. As an illustration of the properties prediction, screenshots corresponding to a calculation for normal hydrogen properties are shown in Fig. 2. In this example, the Leachman [11] equation is selected. The screenshot on the left hand side shows the equation of state selection. The middle one shows the properties to be predicted and the right hand side screenshot is for the calculation output.

\section{2 "All in One Live CD" Application}

In our investigation in the applications of the database, we examined the use of a live $C D$. A live CD application may be an option if the web interface shortcomings are a restrictive consideration. While supporting and maintaining a server there are potential problems, including security related troubles that make it difficult to operate without causing effects to the tasks the users are engaged in. Therefore, we have not started supporting a web server open to the internet other than for providing a web page [13] that furnishes part of the outcome of the present work and the related links to acquire other relevant information on the current project. Besides the disadvantages mentioned above, there may happen a situation that requires to allow only a
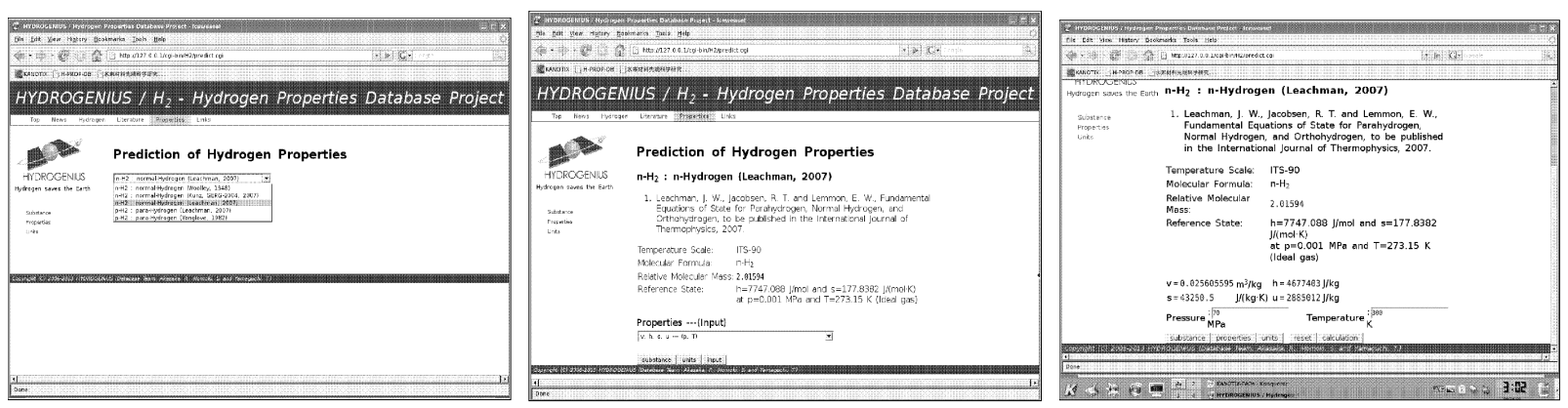

Fig. 2 An example of prediction: calculation of properties of normal hydrogen under the condition of pressure $70 \mathrm{MPa}$ and temperature $300 \mathrm{~K}$ (based on Leachman [11] equation) 
particular group of users to have access to the database. In such cases, a live CD can be used more effectively as an application for the database. A live CD contains operating system (OS) and application software, for example, a web server, web client. A live CD can furnish the OS and application software independent of anything on the hard drive, so even without permanently supporting a server, the database can be used. If access restrictions are a necessary consideration, they can be regulated at the stage of live CD configuration by restricting particular users from certain functions.

The present OS in the "All in One Live CD" is Kanotix [14], a Linux distribution system based on Debian [15]. Kanotix can run as a live CD on practically any computer, automatically detecting and configuring virtually any piece of hardware. The most appealing feature of the "All in One Live CD" application is that it makes the database available not only to the user whose computer is booted from the CD but also to other users as well. Once accessed, the database can act as a calculation server and then database services can be distributed on-line through this server.

\subsubsection{Sharing the Database from a Single Boot}

From a single boot via "All in One Live CD", the database system can run simultaneously on multiple computers, which makes it a unique solution for distribution media. This "All in One Live CD" application can be integrated into an existing local network environment and a single boot from the $C D$ enables users on the local network to share the database.

A computer booted from "All in One Live CD" can act as a server to host our database. For example if a computer, whose IP address is 198.168.1.3, has been booted from the CD as shown in Fig.3. Then the database can be accessed on-line from the other computers by typing http://198.168.1.3 on the address line. The users who do not have the CD at hand are able to acquire the data and to perform the calculations of hydrogen thermophysical properties.

The IP address of the booted computer can be found by using the OS network management function or, if desired, can be setup. For the Kanotix OS we have remastered, there is an easy to use tool "KnetworkManager".

\section{Summary}

As a part of the project undertaken to integrate information on hydrogen thermophysical properties, we have studied database performance requirements and have developed our "All in One Live CD", which can be used as a stand-alone application. This live CD that is bootable and can furnish OS independent of anything on the hard drive. In the present work, the OS is Kanotix [14]. The Kanotix OS is an operating system for live CDs based on a free open source Linux distribution. This OS can run as a live CD on practically any computer, automatically detecting and configuring virtually any piece of hardware. Our "All in One Live CD" can be incorporated into an existing local network environment and a single boot from the $C D$ enables users on the local network to share the database.

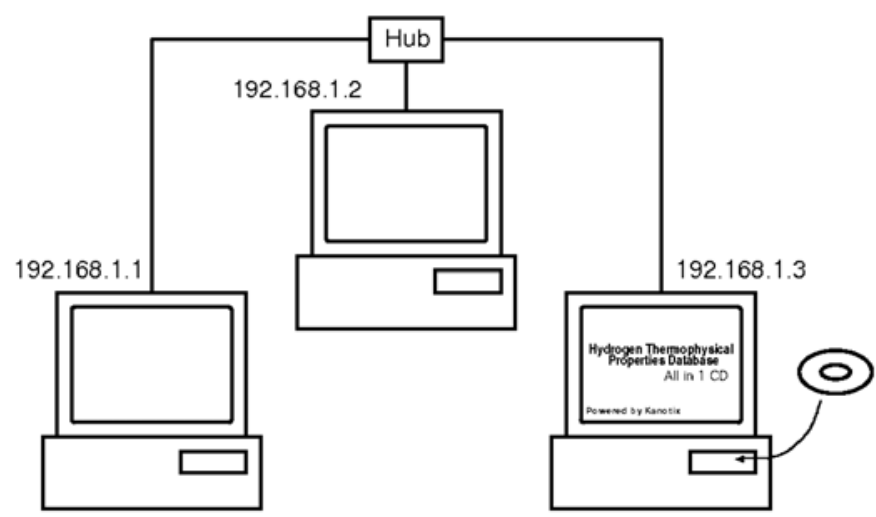

Fig. 3 Booting from "All in One Live CD" makes the database obtainable to all users at the same local network 
The present prediction system proved to be useful for evaluating hydrogen thermophysical properties. The database is not yet available to the public and we are working on improvements and higher levels of development, which will include further experimental data and published information.

\section{Acknowledgments}

This research has been conducted as part of the "Fundamental Research Project on Advanced Hydrogen Science" funded by the New Energy and Industrial Technology Development Organization (NEDO).

\section{References}

1. http://unit.aist.go.jp/hydrogenius/cie/index.html

2. PROPATH GROUP, A PROgram Package for Thermophysical properties of fluids (PROPATH) Version 13.1. (2008) http://gissb.mech.kyushu-u.ac.jp/ akasaka/propath_man/

3. E.W. Lemmon, M.L. Huber, M.O. McLinden, NIST Standard Reference Database 23, NIST Reference Fluid Thermodynamic and Transport Properties Database (REFPROP) Version 8.0. National Institute of Standards and Technology, Standard Reference Data Program, Gaithersburg (2007)

4. NIST Chemistry WebBook, http://webbook.nist.gov/chemistry/

5. N. Sakoda, K. Shindo, K. Shinzato, M. Kohno, Y. Takata and M. Fujii, Int. J. Thermophys. 31, 276 (2010)

6. J. Millat, J.H. Dymond, C.A. Nieto de Castro, Transport Properties of Fluids, ed. by C.A. Nieto de Castro, W.A. Wakeham, Cambridge University Press, 17 (1996)

7. R.T Jacobsen, J.W. Leachman, S.G. Penoncello, W.E. Lemmon, Int. J. Thermophys. 28, 758 (2007)

8. J.W. Leachman, R.T Jacobsen, S.G. Penoncello, M. L. Huber, Int. J. Thermophys. 28, 773 (2007)

9. H.W. Woolley, R.B. Scott, F.C. Brickwedde, J. Res. Natl. Bur. Stand. 41, 379 (1948)

10. O. Kunz, R. Klimech, W. Wagner, and M. Jaeschke, The GERG-2004 Wide-Range Equation of State for Natural Gases and Other Mixtures, GERG Technical Monograph 15 (2007)

11.J. W. Leachman, R.T. Jacobsen, S.G. Penoncello and E.W. Lemmon, J. Phys. Chem. Ref. Data, 38, 721 (2009)

12. B. A. Younglove, J. Phys. Chem. Ref. Data. 11. 1 (1982)

13. http: //h2db. mech.nagasaki-u.ac.jp

14. http://www.kanotix.com

15. http: //www. debian.org 